\title{
INSTABILITY AND ILL-POSEDNESS \\ IN GRANULAR FLOW
}

\author{
By \\ E. Bruce Pitman \\ and \\ David G. Schaeffer
}

IMA Preprint Series \# 511

April 1989 


\title{
INSTABILITY AND ILL-POSEDNESS IN GRANULAR FLOW
}

\author{
E. BRUCE Pitman ${ }^{1}$ \\ DAVID G. SCHAEFFER ${ }^{2}$
}

\begin{abstract}
This paper reviews recent developments in the theory of flowing granular materials. In particular, we highlight new ideas concerning the development of shear bands in these materials.
\end{abstract}

\section{INTRODUCTION}

The deformation and flow of granular materials is a common phenomenon; examples include the flow of coal out of large bunkers and of rice out of a cannister, the yielding of soil under a building and of beach sand under your feet. In spite of the discrete nature of the granular material, researchers often consider it as a continuum for which constitutive relations may be determined. The theory of continuum mechanics is then applied to explain flow behavior.

In this paper, we will review a part of the theory of granular deformation which assumes the material is in plastic yield. In Section 1, we give a brief description of this plasticity theory. Section 2 discusses recent results concerning the mathematical questions of stability and well posedness for the governing equations. Section 3 then examines issues related to the numerical simulation of typical flows.

\section{$\S 1$ PLASTICITY THEORY FOR GRANULAR MEDIA}

In this section, we outline the fundamental equations governing the plastic flow of a granular material. We begin in sub-section (a) by discussing the Critical State Theory $[19,4]$, which models the material as a slightly compressible continuum; a close examination of the Critical State equations is the subject of Section 2. Before that examination, however, we address other issues related to the theory in sub-section (b).

(a) The Critical State Theory. The fundamental equations governing the motion of a continuum are the balance laws for mass and momentum. In fixed Eulerian coordinates these equations take the form:

$$
\begin{gathered}
d_{t} \rho+\rho \partial_{j} v_{j}=0 \\
\rho d_{t} v_{i}+\partial_{j} T_{i j}=0 .
\end{gathered}
$$

Here $\rho$ is the bulk density of the material at the point $x=\left(x_{1}, x_{2}, x_{3}\right)$ and time $t$, $v=\left(v_{1}, v_{2}, v_{3}\right)$ is the velocity, and $T_{i j}$ is the Cauchy stress tensor. We use $d_{t}$ to denote the convective derivative $d_{t}=\partial_{t}+v_{j} \partial_{j}$, and employ the summation convention

\footnotetext{
${ }^{1}$ Supported by the Air Force Office of Scientific Research under grant AFSOR 88-0182, and by the NSF and ONR through grants to the Institute for Mathematics and its Applications

${ }^{2}$ Supported by the National Science Foundation under grant MCS 86-04141.
} 
(where the indices $i, j=1,2,3$ ). It is often important to consider the Lagrangian form of these equations, and to formulate the equations in a properly objective frame of reference. These issues are addressed in the notes of Antman and Trangenstein in these proceedings; in many granular flow applications (for example, flow of material out of a hopper), there is a fixed frame of reference and the Eulerian form of the equations is appropriate.

Equations (1.1) are four equations for the 10 unknowns $\rho, v_{i}, T_{i j}$ (Note: Conservation of angular momentum implies $T$ is symmetric). In order to close the system, we must postulate constitutive laws relating $T$ to $\rho$ and $v$. These relations are: (i) the Yield Condition; (ii) the Flow Rule.

We assume the granular medium is in plastic yield. The Yield Condition specifies a relation among the independent elements of $T$ and the density:

$$
\Phi(T, \rho)=0 .
$$

For fixed $\rho$, this equation defines a closed surface in stress space. In the interior of this surface material would behave elastically; we make the rigid-plastic assumption which means deformation may occur only for stresses on the surface. We fix a sign convention by regarding compressive stresses as positive. (Remark: For some materials, $\Phi$ may be taken to have the form

$$
\Phi(T, \rho)=\phi(\operatorname{tr}(T),|\operatorname{dev}(T)|)-\rho^{\frac{2}{\beta}} .
$$

Here, $\operatorname{tr}(T)$ denotes the trace of $T ;|\operatorname{dev}(T)|$ is the Euclidean norm of the deviator of $T$, where

$$
\operatorname{dev}(T)=T-\frac{\operatorname{tr}(T)}{3} I
$$

and $\beta$ is a small parameter which measures compressibility, typically $\beta \approx 10^{-1}-10^{-2}$.)

It is customary to assume that $\Phi(\cdot, \rho)$ is a strictly convex function on $R^{6}$. We also assume that $\Phi$ is monotone in $\rho$; this monotonicity generates a nested sequence of yield surfaces in stress space, indexed by the density. Such a picture lies at the heart of the Critical State theory.

We adopt an Associative Flow Rule which assumes that the normal $\frac{\partial \Phi}{\partial T}$ and the strain rate tensor $V$ are proportional:

$$
\frac{\partial \Phi}{\partial T_{i j}}=\mu V_{i j}
$$

Here $V_{i j}=-\frac{1}{2}\left(\partial_{i} v_{j}+\partial_{j} v_{i}\right)$. In Equation (1.3), $\mu$ is a positive scalar function, but is not specified a priori (as in elasticity). Rather, $\mu$ must be determined as part of the solution procedure.

We claim that the system of equations $(1.1,1.2,1.3)$ is closed. There are now 11 unknowns, $\mu$ being added to the previous list; there are also 11 equations with which to determine these variables. However, Equations $(1.2,1.3)$ are not evolution equations, and the mixed differential/algebraic character makes the system difficult to analyze. Considerable simplification results if we solve $(1.2,1.3)$ for $T$ and $\mu$ as functions of $V$ and $\rho$ and write

$$
T=T(V, \rho)
$$


We remark that in (1.4), $T$, when considered as a function of $V$, is homogeneous of degree zero; this homogeneity is characteristic of a rate independent theory. We may substitute (1.4) into (1.1) to obtain a system of four time dependent partial differential equations in

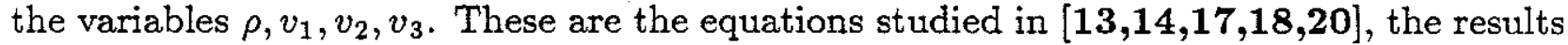
of which are reviewed in Section 2. In the next sub-section we discuss the limitations of and alternatives to the Critical State theory.

(b) Alternative Theories. For expositional clarity, it is useful to restrict attention to two dimensions in this sub-section. Then $T$ is a $2 \times 2$ matrix with eigenvalues $\sigma_{1} \geq \sigma_{2}$. We begin by reformulating the Critical State equations in this setting, writing $u$ and $v$ for the $x$ and $y$ components of the velocity, respectively [4].

Define the mean stress $\sigma$ and the shear stress $\tau$

$$
\sigma=\frac{\sigma_{1}+\sigma_{2}}{2} \quad \tau=\frac{\sigma_{1}-\sigma_{2}}{2} .
$$

The yield loci are then defined by a relation $\tau=f(\sigma, \rho)$; the self-similarity of these loci implies that the "Critical States", defined by $\tau_{\sigma}=0$, lie along a common line $\tau=\sigma \sin (\delta)$, where the material parameter $\delta$ is called the angle of internal friction. The Critical State divides the yield locus into the consolidation (or hardening) side with $\tau_{\sigma}<0$ and the expansion (or softening) side with $\tau_{\sigma}>0$. We follow $[4,5]$ and perform a non-linear change of stress variables to obtain

$$
T=\sigma\left(\begin{array}{ll}
1 & 0 \\
0 & 1
\end{array}\right)+\tau\left(\begin{array}{rr}
\cos (2 \psi) & \sin (2 \psi) \\
\sin (2 \psi) & -\cos (2 \psi)
\end{array}\right)
$$

Here, $\psi$ is the angle between the axis of major principal stress (i.e., the eigenvector associated with the eigenvalue $\sigma_{1}$ ) and the $x$-axis. In these coordinates the flow rule may be shown to take the form

$$
\begin{aligned}
\sin (2 \psi)\left(\partial_{x} u-\partial_{y} v\right) & =\cos (2 \psi)\left(\partial_{y} u+\partial_{x} v\right) \\
\tau_{\sigma}\left(\partial_{x} u-\partial_{y} v\right) & =\cos (2 \psi)\left(\partial_{y} v+\partial_{x} u\right) .
\end{aligned}
$$

Using (1.5) in (1.1b), the continuity and momentum equations take their usual form. We note that, while (1.1) are time-dependent conservation laws, the flow rule (1.6) are steadystate $\mathrm{PDE}$ and are not conservation laws.

Of particular concern to the engineering community is the steady-state system which results from dropping the $d_{t}$ terms from (1.1). In the $(x, y)$-plane, these steady-state equations constitute a first order hyperbolic system for the variables $(\rho, u, v, \sigma, \psi)$. The characteristics are $(i)$ streamlines of the velocity field; $(i i)$ two pair of coincident directions making an angle $\pm \alpha$ with the axis of major principal stress, where

$$
\tan (\alpha)=\sqrt{\frac{1-\tau_{\sigma}}{1+\tau_{\sigma}}}
$$


There are, however, only three eigenvectors for this system. This eigenvector deficiency precludes analysis via the standard hyperbolic methods.

An even simpler system results from assuming the material is incompressible. Then $\rho$ is constant, and (1.1a) states that the velocity field is divergence-free; from equation (1.6b), the stress field must be at the critical state $\tau_{\sigma}=0$ and the shear stress is given by $\tau=\sigma \sin (\delta)$. The reduced system of four equations for $(u, v, \sigma, \psi)$ is strictly hyperbolic, as the double characteristics (1.7) split into four distinct characteristic directions $[5,12]$. This incompressible limit of the compressible equations is highly singular, and it is not known how solutions of the two systems are related.

The Associative Flow Rule (1.3) is challenged by many authors; indeed, the experiments of Drescher [2] show evidence for misalignment of $T$ and $V$ under some conditions. deJosselin de Jong [9] and Mehrabadi and Cowin [11], among others, have proposed nonAssociative theories appropriate for two dimensional flows. Spencer [21] has developed a theory which may be formulated in either two or three dimensions; he assumes the material is incompressible, and adds to the alignment condition (1.6a) terms involving derivitives of $\psi$. These terms account for local rotations of a material element during flow. The resulting steady state equations are again hyperbolic, with two pair of coincident characteristic directions. The question of which flow rule is "correct" is not resolved however, since experimental measurements are difficult and are currently unable to distinguish among the many alternatives.

Regarding the evolution equations for an incompressible material, Schaeffer [16] examined a rigid-plastic model that postulated the alignment of $V$ and $\operatorname{dev}(T)$. His principle conclusion was that the equations are prone to violent instability, similar to that of the mixed forward-backward heat equation

$$
\partial_{t} u=\partial_{x x} u-\partial_{y y} u .
$$

More precisely, for a wedge of directions in Fourier Transform space, exponential solutions of the linearized equations exhibit uncontrolled growth, proportional to $O\left(|\xi|^{2}\right)$ where $\xi$ is the Fourier Transform variable. As we shall see in the next section, compressibility effects mollify this instability. (Remark: The evolution equations given by Spencer's theory may best be characterized as elliptic. See [16] for details.)

The framework of incompressible plasticity for granular materials was developed from the plasticity theory for metals. The metal is considered incompressible, and the yield condition is assumed to be independent of the mean stress, often taking the form $|\operatorname{dev}(T)|=\kappa$ where $\kappa$ is a constant. An Associative Flow Rule is used. The metal equations are not prone to the instabilities which plague granular flows. By the late 1950's, it was recognized that the particulate nature of granular materials allowed for density variations during deformation. Grigorian [3] proposed a simple density dependent theory in 1960, and Roscoe [15] was developing the ideas which led to the Critical State Theory around the same time. We now turn to an examination of the Critical State equations.

\section{$\S 2$ ILL POSEDNESS AND INSTABILITY IN THE CRITICAL STATE THEORY}

We first review some results concerning the well posedness of the initial value problem for the equations $(1.1,1.4)$. The second sub-section examines questions of stability. 
(a) Ill Posedness. Substituting (1.4) into (1.1b), the equations of motion may be written

$$
\begin{gathered}
d_{t} \rho+\rho \partial_{j} v_{j}=0 \\
\rho d_{t} v_{i}+\left(\frac{\partial T_{i j}}{\partial \rho}\right) \partial_{j} \rho-\left(\frac{\partial T_{i j}}{\partial V_{k l}}\right) \partial_{j} \partial_{k} v_{l}=0
\end{gathered}
$$

Equations (2.1) formally resemble the Navier-Stokes equations for a viscous compressible fluid. However, this apparent similarity is illusory. The primary difference between the fluid equations and (2.1) is in the nature of the viscous dissipation term $\frac{\partial T}{\partial V}$. In the Navier-Stokes system, the symbol matrix associated to this dissipation is positive-definite; in (2.1), the symbol is only semi-definite. The non-definiteness of this symbol is the source of instabilities in granular flows. This section will explain how these instabilities arise.

Introduce the principal strain rates $\epsilon_{i}$, the the eigenvalues of $V$. Our constitutive relations assume that material is actually deforming, so not all the $\epsilon_{i}$ can be zero simultaneously. We may think of (2.1) in either two or three dimensions, although it is often easier to work with $(1.1,1.6)$ for deformations in two dimensions.

We begin by defining the principal symbol associated with (2.1). To this end, introduce weights as in [1]; specifically, assign the weights $s_{k}=2, k=1,2,3,4$ to the 4 equations $(2.1)$, and $t_{1}=t_{2}=t_{3}=0, t_{4}=1$ to the variables $v_{i}, \rho$, respectively. With these weights, the contribution of the $m^{\text {th }}$ unknown to the principle part in the $k^{\text {th }}$ equation consists of all derivatives of the order $s_{k}-t_{m}$, i.e., all second derivatives of $v$ and first derivatives of $\rho$. The system is then quasi-linear in that the highest order derivatives appear linearly, with coefficients which are of lower order. Linearize the problem by "freezing" the coefficients and looking for exponential solutions of the form

$$
\left[\begin{array}{l}
\rho \\
v
\end{array}\right]=\exp (i(x, \xi)+\lambda t)\left[\begin{array}{l}
\bar{\rho} \\
\bar{v}
\end{array}\right]
$$

where $(x, \xi)=x_{i} \xi_{i}$. This linearization generates an eigenvalue problem for $\lambda$

$$
S(\xi)\left[\begin{array}{l}
\bar{\rho} \\
\bar{v}
\end{array}\right]=\lambda I\left[\begin{array}{l}
\bar{\rho} \\
\bar{v}
\end{array}\right]
$$

with the principal part of $S(\xi)$ given in block form by

$$
\left(\begin{array}{cc}
i(\xi, v) & 0 \\
q(\xi) & Q(\xi)
\end{array}\right)
$$

In this equation $I$ is the identity matrix, $q(\xi)$ is the column vector defined by

$$
q_{i}(\xi)=-i\left(\frac{\partial T_{i j}}{\partial \rho}\right) \xi_{j}
$$

and $Q$ is the matrix whose $(i, k)$-entry is

$$
Q_{i k}(\xi)=-\left(\frac{\partial T_{i j}}{\partial V_{k l}}\right) \xi_{j} \xi_{l}
$$


In either two or three dimensions, we will call the system of equations (2.1) (linearly) well posed if for each eigenvalue $\lambda_{n}$ of (2.3), the real part is bounded:

$$
\sup _{\xi} \operatorname{Re} \lambda_{n}(\xi)<\infty
$$

we will say the system is (linearly) stable if each eigenvalue is negative

$$
\sup _{\xi} \operatorname{Re} \lambda_{n}(\xi)<0
$$

Schaeffer and Pitman $[\mathbf{1 3}, \mathbf{1 7}]$ examined the well posedness of $(2.1)$ in two and three dimensions. Those results may be summarized by the following

THEOREM 2.1. The system (2.1) is well posed in three dimensions if all three strain rates $\epsilon_{i}, i=1,2,3$ are non-zero. If one of the strain-rates is zero (e.g. the two dimensional theory, in which case one of the strain-rates is always zero, or the three dimensional theory with, say, translation invariance), the system remains well posed unless $1 \geq \tau_{\sigma} \geq 0$.

The underlying reason for the difference between two and three dimensional flow is the structure of $Q$. If, in three dimensions all the $\epsilon_{i}$ are non-zero, then $Q$ is negative-definite. The eigenvalues of the principal part of $S$ are the one pure imaginary entry $i(x, \xi)$ and the three negative eigenvalues of $Q$, and a simple perturbation theory argument establishes similar bounds for the eigenvalues of the full symbol. However in two dimensions, the matrix corresponding to $Q$ is only semi-definite. Along two distinguished lines in Fourier Transform space, namely the (normals to the) characteristics (1.7) of the steady-state equations, one of the eigenvalues of $Q$ vanishes. Higher order terms are required to complete the analysis, and the next term of the perturbation expansion (which includes $q$ ) may be of either sign. More specifically, along the normals to the characteristics, one of the eigenvalues of $Q$ is $O\left(-|\xi|^{2}\right)$ while the other eigenvalue is $\tau_{\sigma} O(|\xi|)$. Hence (2.1) is ill posed on the expansion side of the yield locus. We note that the ill posedness of the Critical State equations is less catastrophic than the ill posedness Schaeffer discovered for the incompressible equations.

If one assumes a non-Associative Flow Rule, the two lines of degeneracy open into two wedges. In the interior of these wedges, the equations of motion are ill posed, with a growth rate of $O\left(|\xi|^{2}\right)$. Elasticity effects can close the wedges; see Mandel [10].

(b) Instability. For many typical granular flows, there exist two well separated time scales [13]. It is possible to exploit these separate scales and incorporate into our analysis additional terms of the symbol $S$. To this end, define a new time $\tilde{t}=\epsilon t$ and a similarly scaled velocity $\tilde{v}=\epsilon^{-1} v$. Substitute into (2.1) and recall the homogeneity of $T(V, \rho)$ to find (dropping the tilde)

$$
\begin{gathered}
\partial_{t} \rho+\partial_{j}\left(\rho v_{j}\right)=0 \\
\epsilon^{2} \rho d_{t} v_{i}+\left(\frac{\partial T_{i j}}{\partial \rho}\right) \partial_{j} \rho-\left(\frac{\partial T_{i j}}{\partial V_{k l}}\right) \partial_{j} \partial_{k} v_{l}=0
\end{gathered}
$$


Now make the quasi-dynamic approximation by setting $\epsilon=0$. Linearize the equations about a steady, homogeneous solution $\rho_{0}, v_{0}$. (In the linearization, four terms arise from the derivitive $\partial_{j}\left(\rho v_{j}\right)$; we will drop all of these terms except $\rho_{0} \partial_{j} v_{j}$. See [18] for details.) Again looking for exponential solutions in the form (2.2), we derive a generalized eigenvalue problem

$$
S(\xi)\left[\begin{array}{l}
\bar{\rho} \\
\bar{v}
\end{array}\right]=\left[\begin{array}{c}
\lambda \bar{\rho} \\
0
\end{array}\right] .
$$

Here the principal part of $S(\xi)$ has the block structure

$$
\left(\begin{array}{cc}
0 & \xi^{*} \\
-q(\xi) & Q(\xi)
\end{array}\right)
$$

where $\xi^{*}$ denotes the transpose of the column vector $\xi$. Performing a similarity transformation to eliminate complex entries, $q(\xi)$ takes the form

$$
q_{i}(\xi)=\rho_{0}\left(\frac{\partial T_{i j}}{\partial \rho}\right) \xi_{j}
$$

and $Q$ remains as in (2.6).

In two dimensions, our stability results relate the growth of the generalized eigenvalue $\lambda$ in (2.8) to the ellipticity of the steady state equations associated to (2.7)

$$
\begin{gathered}
\rho_{0} \partial_{j} v_{j}=0 \\
\left(\frac{\partial T_{i j}}{\partial \rho}\right) \partial_{j} \rho-\left(\frac{\partial T_{i j}}{\partial V_{k l}}\right) \partial_{j} \partial_{k} v_{l}=0
\end{gathered}
$$

Call (2.9) elliptic if $\operatorname{det} S(\xi) \neq 0$ for all nonzero $\xi \in R^{2}$. Roughly speaking, our first result states that (2.7) become unstable when (2.9) change type from elliptic to hyperbolic.

THEOREM 2.2. In two dimensions, the system (2.7) is stable if and only if (i) Equation (2.9) is elliptic, and (ii) $\sigma_{i} \geq 0, i=1,2$.

One implication of this theorem is that, even for some stresses on the consolidation side of the yield locus, (2.7) is unstable. This statement contradicts a widely held view that deformation is stable on the consolidation side of the yield locus and unstable on the expansion side; Theorem 2.1 shows that the Critical State is the boundary between well posed and ill posed behavior. A second consequence of the theorem is that all Fourier modes along a specific direction become unstable simultaneously. Which specific mode eventually dominates growth (in the linear regime) depends on initial and boundary conditions; this suggests extreme sensitivity of experiments to imperfections, as is observed in the laboratory.

The analogue of Theorem 2.2 holds in three dimensions, but the geometric interpretation is more complicated. In particular, when the steady state equations lose ellipticity they do not become hyperbolic; rather they are of mixed type. 


\section{$\S 3$ SHEAR BANDS AND NUMERICAL SIMULATIONS}

The analytic investigations of the previous section considered only linearized equations. Other techniques, particularly numerical computations, need to be employed on the nonlinear equations. Two different flow regimes are of particular importance. The first is slow flow in a hopper. The separation of time scales exploited in the quasi-dynamic approximation can be used, and one need only solve (2.7) with $\epsilon=0$, rather than the full equations (2.1). We note the structure of this system: a single time dependent hyperbolic equation for the density coupled to (hopefully!) an elliptic system for the velocities. Experience with the equations for two phase flow in a porous medium should be helpful in developing computational strategy for this problem.

The second flow to be investigated is shear flow. Consider the following scenario suggested by Theorems 2.1 and 2.2 for the development of shear bands. Beginning with a homogeneous stable flow, material consolidates upon deformation, eventually reaching the instability locus. Subsequent deformation remains smooth, but it is no longer homogeneous and local imperfections grow in time. At some point in the sample, an imperfection ultimately becomes ill posed as it reaches the Critical State locus. Such a point could act as the seed of a velocity discontinuity which then expands into a shear band. Numerical simulations should be able to confirm this conjectured route to shear band formation, but the ill posedness of two dimensional deformations precludes the simple intergration of Equations (2.1). We follow a suggestion of Jackson and regularize the system.

Let us elaborate. Near a shear band, there is large momentum transfer over distances on the order of a particle diameter. Jenkins [6], Savage [7], and colleagues have developed a theory for granular flow at very high shear rates based on an extension of standard kinetic theory to slightly inelastic particles. Incorporating the Enskog dense gas correction, the granular continuum equations are essentially the Navier-Stokes equations for a compressible, heat conducting fluid. The "heat" is not molecular energy, but the so-called "granular temperature" measuring a particle's deviation from the mean motion of its neighbors. There is one non-classical term in the theory, a heat sink which represents the energy loss due to inelastic collisions. Let the stress tensor derived from this kinetic theory be $T^{k}$, and denote the stress tensor of the Critical State theory as $T^{f}$. Jackson [8] considers a system consisting of (1.1) and the energy equation of the kinetic theory, with the total stress being the sum

$$
T=T^{f}+T^{k}
$$

[8] also shows how to derive appropriate boundary conditions for flow.

Let us apply Jackson's ideas to the following two dimensional experiment. Consider a shear cell filled with small beads. Initially hold the top and bottom plates of the cell at rest; apply periodic boundary conditions at the left and right ends and appropriate conditions at the plates. Assume gravity is acting in the vertical direction, and at time zero drive the bottom plate at a prescribed speed. [8] analyzes a one dimensional steady state version of these equations, using an ad hoc formulation of $T^{f}$.

In a full simulation of the equations, attention must be given to two details. First, dynamics on all time scales must be adequately resolved. In particular, interactions over 
time steps of $O(\epsilon)$ (in the notation of Section 2b) must be analyzed; such detail necessitates a large expenditure of computational effort. The second important detail is to note the small diffusion present in the system. Along the degenerate directions (1.7), the Critical State terms $T^{f}$ contain no dissipation; when non-dimensionalized, the kinetic terms $T^{k}$ are scaled by a small parameter $\eta^{2}$, where $\eta$ is the ratio of particle diameter to the gap width of the cell. Hence, at least near the degenerate lines, the system is dominated by hyperbolic effects. The numerical method employed to integrate the equations must not introduce artificial viscosity which will degrade resolution along these directions.

The figure below shows suggestive results from a preliminary time dependent calculation in one space dimension using the regularized equations (3.1). Operator splitting is employed. An upwind-Godunov scheme is used for the hyperbolic terms, and an explicit three-level scheme is employed on the diffusion terms. No slip boundary conditions are assumed on the top and bottom plates. The figure shows the long-time asymptotic behavior of the horizontal velocity $\left(\times \frac{1}{10} \frac{m}{s e c}\right.$, plotted along the abscissa) versus non-dimensional height in the cell (the ordinant), for two different values of the driving plate speed. When shearing the sample at high speed, the velocity profile is almost linear. At low driving speed, the bottom half of the cell moves almost a plug, with shearing confined to the upper half of the cell. These figures are in approximate agreement with experimental observations (see [8] for references) of shear band formation in flowing granular materials.
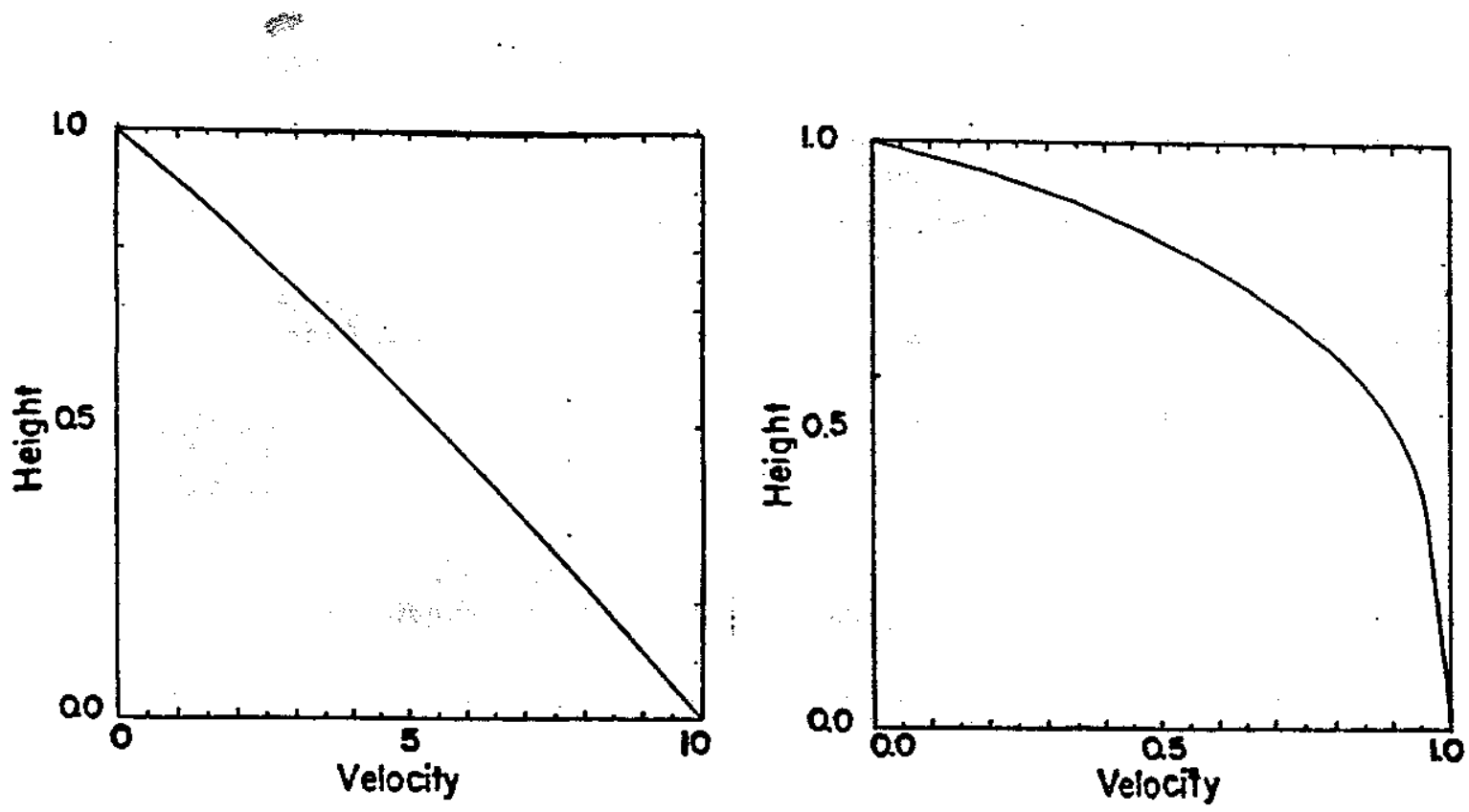


\section{REFERENCES}

1. S. Agmon, A. Douglis, and L. Nirenberg, Estimates Near the Boundary for Solutions of Elliptic Partial Differential Equations Satisfying General Boundary Conditions II, Comm. Pure Appl. Math 17 (1964), 35-92.

2. A. Drescher, An Experimental Investigation of Flow Rules for Granular Materials using Optically Sensitive Glass Particles, Geotech. 26 (1976), 591-601.

3. S. S. Grigorian, On Basic Concepts in Soil Dynamics, P.M.M. (Soviet Appl. Math. Mech.) 24 (1960), 1057-1072.

4. R. Jackson, Some Mathematical and Physical Aspects of Continuum Models for the Motion of Granular Materials, in "The Theory of Dispersed Multiphase Flow," R. Meyer (ed.), Academic Press (1983).

5. A. W. Jenike, Steady Gravity Flow of Frictional-Cohesive Solids in Converging Channels, J. Appl. Mech. 31 (1964), 5-11.

6. J. T. Jenkins and M. W. Richman, Grad's 13 Moment System for a Dense Gas of Inelastic Spheres, Arch. Rat'l. Mech. Anal. 87 (1985), 355-377.

7. J. T. Jenkins and S. B. Savage, A Theory for the Rapid Flow of Identical, Smooth, Nearly Elastic Spherical Particles, J. F. M. 130 (1983), 187-202.

8. P. C. Johnson and R. Jackson, Frictional-Collisional Constitutive Relations for Granular Materials, J. F. M. 176 (1987), 67-93.

9. G. deJosselin deJong, The Double Sliding Free Rotating Model for Granular Assemblies, Geotech. 21 (1971), 155-163.

10. J. Mandel, Conditions de Stabilite et Postulat de Drucker, in 'Int'l Symposium on Rheology and Soil Mechanics," Grenoble, IUTAM (1964).

11. M. M. Mehrabadi and S. C. Cowin, Initial Planar Deformation of Dilitant Granular Materials, J. Mech. Phys. Solids 26 (1978), 269-284.

12. E. B. Pitman, The Stability of Granular Flow in Converging Hoppers, SIAM 3. Appl. Math. 48 (1988), 1033-1053.

13. E. B. Pitman and D. G. Schaeffer, Stability of Time Dependent Compressible Granular Flow in Two Dimensions, Comm. Pure Appl. Math. 40 (1987), 421-447.

14. E. B. Pitman, D. G. Schaeffer and M. Shearer, Stability in Three Dimensional Critical State Theories of Plasticity, in preparation.

15. K. H. Roscoe, A. N. Schofield and C. P. Wroth, On the Yielding of Soils, Geotech. 8 (1958), 22-53.

16. D. G. Schaeffer, Instability in the Evolution Equations Describing Incompressible Granular Flow, J. Diff. Eq. 66 (1987), 19-50.

17. D. G. Schaeffer and E. B. Pitman, Ill Posedness in Three Dimensional Plastic Flow, to appear, Comm. Pure Appl. Math..

18. D. G. Schaeffer, M. Shearer and E. B. Pitman, Instability in Critical State Theories of Granular Flow, preprint.

19. A. N. Schofield and C. P. Wroth, "Critical State Soil Mechanics," McGraw-Hill Publishing (1968).

20. M. Shearer and D. G. Schaeffer, Foundations of the Quasi-dynamic Approximation in Critical State Plasticity, preprint.

21. A. J. M. Spencer, Deformation of Ideal Granular Materials, in "Mechanics of Solids," H. Hopkins and M. Sewell (eds.), Pergamon Press (1982).

Pitman: Institute for Mathematics and its Applications University of Minnesota Minneapolis, Minnesota 55455 and Department of Mathematics New Jersey Institute of Technology Newark, New Jersey 07102; Schaeffer: Department of Mathematics Duke University Durham, North Carolina 27706 